\title{
MANAGEMENT OF ANTERIOR SHOULDER INSTABILITY WITH SEVERE GLENOID BONE LOSS (> 25\%) TREATED BY OPEN LATARJET PROCEDURE- CASE SERIES
}

\author{
Vijayaragavan Pugalenthi', Rathinam Arivasan², Thanga Muthu Saravana Muthu³, Rama Krishnan Muthusamy4
}

1 Professor and HOD, Department of Orthopaedics, Madurai Medical College, Madurai, Tamilnadu.

${ }_{2}^{2}$ Professor, Department of Orthopaedics, Madurai Medical College, Madurai, Tamilnadu.

${ }^{3}$ Assistant Professor, Department of Orthopaedics, Madurai Medical College, Madurai, Tamilnadu.

${ }_{4}^{4}$ Postgraduate Student, Department of Orthopaedics and Traumatology, Madurai Medical College, Madurai, Tamilnadu.

\section{ABSTRACT}

\section{BACKGROUND}

Arthroscopic management of glenohumeral instability with Bony Bankart's lesion ( $>25 \%$ bone loss) have been associated with high rates of recurrence in glenohumeral osseous defects. In such patients, Latarjet procedure can be considered as better solution.

\section{MATERIALS AND METHODS}

Total 10 patients were studied. Bony defect of the anterior glenoid ( $>25 \%$ ) with three or more previous dislocation were included. Latarjet operation makes use of large coracoid bone graft to extend glenoid articular surface by means of lengthened bone platform, dynamic stability is provided by conjoint tendon, static stability given by coracoacromial ligament. Constant-Murley score was used to evaluate results.

Postoperative Management- Immobilisation in sling for first 6 weeks with limited abduction, flexion and external rotation. Antiinflammatory pain medications were avoided in early postoperative period to optimise osseous healing. Active strengthening is permitted once osseous healing is visualised.

\section{RESULTS}

Osseous union of graft was achieved in all patients. There was no recurrence of instability. Average follow-up period was 24 months. Average forward flexion was 160 and external rotation was 42.2. Mean postoperative Constant-Murley score was 80 . Abduction and external rotation were minimally reduced. 2 patients had post-op superficial wound infection, which was treated with IV antibiotics.

\section{CONCLUSION}

Open Latarjet reconstruction can successfully restore shoulder stability and range of movements in joints with a significant bony defect of the glenoid even in elderly patients. It is effective in situations in which soft-tissue reconstruction is not a reasonable option.

\section{KEYWORDS}

Bone Loss; Bony Bankart's, Latarjet Procedure, Conjoint Tendon, Recurrent Shoulder.

HOW TO CITE THIS ARTICLE: Pugalenthi V, Arivasan R, Muthu TMS, et al. Management of anterior shoulder instability with severe glenoid bone loss (> 25\%) treated by open Latarjet procedure- case series. J. Evolution Med. Dent. Sci. 2018;7(18): 22932297, DOI: $10.14260 /$ jemds/2018/516

\section{BACKGROUND}

Recurrent shoulder dislocations are common in physically active young adult males who are participating in sports activities (Contact sports like rugby, kabbadi, basketball and hockey).

The disruption between the anterior-inferior labrum and the glenoid was termed the "essential lesion" by Bankart in 1938. And if the IGHLC detaches with a small piece of avulsed glenoid, the lesion is called a bony Bankart lesion.

Normally, in abduction and external rotation, humeral head gives compressive load to the antero-inferior part of glenoid. It is evenly distributed to bony and soft tissue component. If bony glenoid lesion of $>25 \%$ are treated by soft tissue procedures, the compressive load given by the

'Financial or Other Competing Interest': None.

Submission 28-02-2018, Peer Review 12-04-2018,

Acceptance 18-04-2018, Published 30-04-2018.

Corresponding Author:

Muthu Samy Rama Krishnan,

2/537, Sellandy Nagar, Kaitiganapalli,

Krishnagiri District-635002, Tamilnadu.

E-mail: drmuthu222@gmail.com

DOI: $10.14260 /$ jemds $/ 2018 / 516$ humerus is maximum at bone and soft tissue interface. This causes the failure of repair. Typically, glenoid bone loss of $25 \%$ of the surface area of the glenoid is an indication for bony reconstruction of this defect, which most commonly occurs antero-inferiorly.

Different bone augmentation techniques including the Latarjet procedure, iliac crest autograft or structural allograft are practised with their own advantage and disadvantages.

The Latarjet procedure involving transfer of the coracoid process to the antero-inferior glenoid has been the most well studied and popular of these techniques.

Best method to correct this bone loss is by open Latarjet procedures. Advantages of this procedures are increase in the glenoid surface area by autogenous coracoids graft, static stability by coracoacromial ligament which is sutured with the capsule, dynamic stability by conjoint tendon.

Boileau P et al1- The higher failure rates reported with arthroscopic stabilisation of traumatic and recurrent anterior shoulder instability compared with open stabilisation remain a concern.

\section{Aims and Objectives}

To assess the functional outcome of Latarjet procedure in patients with recurrent shoulder dislocation with Bony 
Bankart's lesion and to provide pain-free mobile shoulder without instability.

\section{MATERIALS AND METHODS \\ Design- Case Series.}

Period- August 2015 to September 2017.

\section{Sample Size}

10 cases were taken up for our study. There were 7 male and 3 female patients, ranging in age from $24-60$ years with the mean age of 28 years.

The right shoulder was involved in 6 patients $(60 \%)$ and the left shoulder was involved in 4 patients (40\%).

\section{Inclusion Criteria}

1. Patients with recurrent shoulder dislocations, more than 2 to 3 episodes.

2. Patients with bony Bankart's lesion $>25 \%$ bone loss.

3. Age between $18-60$ yrs.

4. Skeletally mature patients of both sexes.

5. Minimum of 24 months follow-up.

\section{Exclusion Criteria}

1. Comorbid conditions not permitting major surgical procedures.

2. Poor local skin conditions.

3. Patients with a concomitant rotator cuff lesion.

4. An acute first-time dislocation.

5. Surgery for a painful, unstable shoulder without true dislocation or subluxation.

6. Multidirectional instability.

Patients with recurrent shoulder dislocation with Bony Bankart's lesion admitted at Govt. Rajaji Hospital in the Dept. of Orthopaedics and Traumatology, Madurai were taken up for study after obtaining informed consent.

\section{Preoperative Workup}

Patients underwent a preoperative evaluation including the following parameters- $\mathrm{Hb}$, blood sugar, ECG, RFT, x-ray chest in order to get fitness for surgery.

\section{X-Ray Chest with both Shoulders}

It will identify acromioclavicular disruptions, associated fractures of clavicle, humerus, scapula and chest injury.

- Axillary View.

- $\quad$ Stryker Notch View.

- The Grashey View.

- The West Point Axillary.

- The Scapular Y View.

- The Apical Oblique View.

\section{Computed Tomography}

Bishop JY et al $^{2}$ concluded three-dimensional CT was the most reliable imaging modality for predicting glenoid bone loss. Regular CT was the second most reliable and reproducible modality.

\section{Magnetic Resonance Imaging}

If consideration is given to surgical treatment, MRI (in comparison to $\mathrm{CT}$ ) is considered the standard reference for the determination of pathoanatomy because the majority of injuries are capsuloligamentous. MRI is also necessary to evaluate for rotator cuff tears and humeral avulsions of the glenohumeral ligaments (HAGL).

\section{Calculation of Glenoid Bone Loss}

A best-fit circle of the inferior two-thirds of the normal shoulder can be superimposed onto the effected side to estimate the amount of bone loss. The average diameter of this circle for a normal glenoid is approximately $24 \mathrm{~mm}$.

An anterior defect of $7.5 \mathrm{~mm}$ corresponds to approximately $25 \%$ of total bone loss.

Itoi $\mathrm{E}$, Lee $\mathrm{SB}$ et $\mathrm{al}^{3}$ reported an osseous defect with a width that is at least 21 percent of the glenoid length that may cause instability and limit the range of motion of the shoulder after Bankart repair.

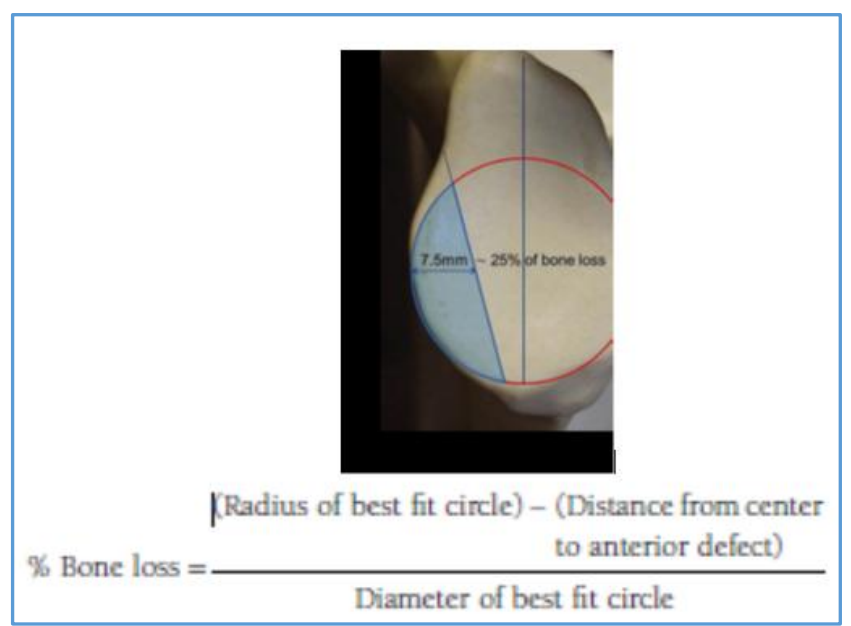

\section{Anaesthesia}

General anaesthesia (or) interscalene block with paravertebral block.

Positioning- Beach-chair position was used.

\section{Surgical Approach}

An open deltopectoral approach, an initial arthroscopic evaluation of the glenohumeral joint to confirm or determine that bony reconstruction of a glenoid and/or Hill-Sachs defect was done.

\section{Procedure}

Graft Harvesting

(Initial Step).

\section{Step 1}

Through standard deltopectoral approach coracoid is exposed for osteotomy.

\section{Step 2}

Pectoralis minor tendon released from the medial aspect of the coracoid and the bone is exposed proximally to its base.

\section{Step 3}

The coracoacromial ligament is then released, leaving a 1-cm stump of the ligament attached to the coracoid process laterally. 


\section{Step 4}

The conjoined tendon attached to the osteotomised coracoid, is mobilised. Care is taken not to injure the musculocutaneous nerve during mobilisation.

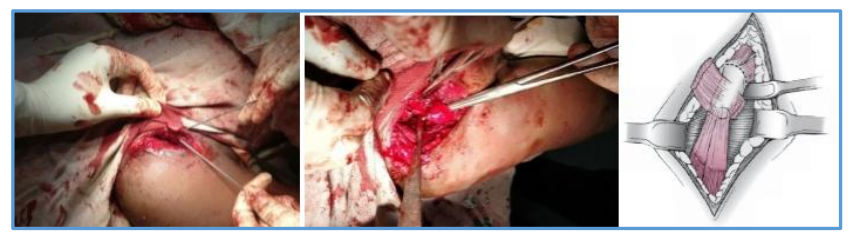

\section{Graft Fixation}

Step 1

The glenohumeral joint exposed similar to an open Bankart repair.

\section{Step 2}

The glenoid rim, neck in the area of the defect and the coracoid surface that will be placed into the defect site are debrided of any soft tissue and lightly decorticated with a high-speed burr to establish a bed of healthy, bleeding bone.

\section{Step 3}

Superior and inferior drill holes are made through the coracoid graft.

\section{Step 4}

Coracoid graft is placed in the glenoid defect below the glenoid equator, flush with the glenoid rim. The position held with temporary K-wires.

\section{Step 5}

The superior hole in the coracoid is then used to drill through the glenoid bicortically followed by screw placement. The same steps are repeated to place the inferior screw.

\section{Step 6}

Partially threaded ( 30 to $36 \mathrm{~mm}$ ), $4.0 \mathrm{~mm}$ cancellous or 3.5 $\mathrm{mm}$ cortical screws used for coracoid fixation in a "lag" fashion.

\section{Step 7}

The lateral capsular flap made during capsular incision can then be repaired to the coracoacromial ligament stump.

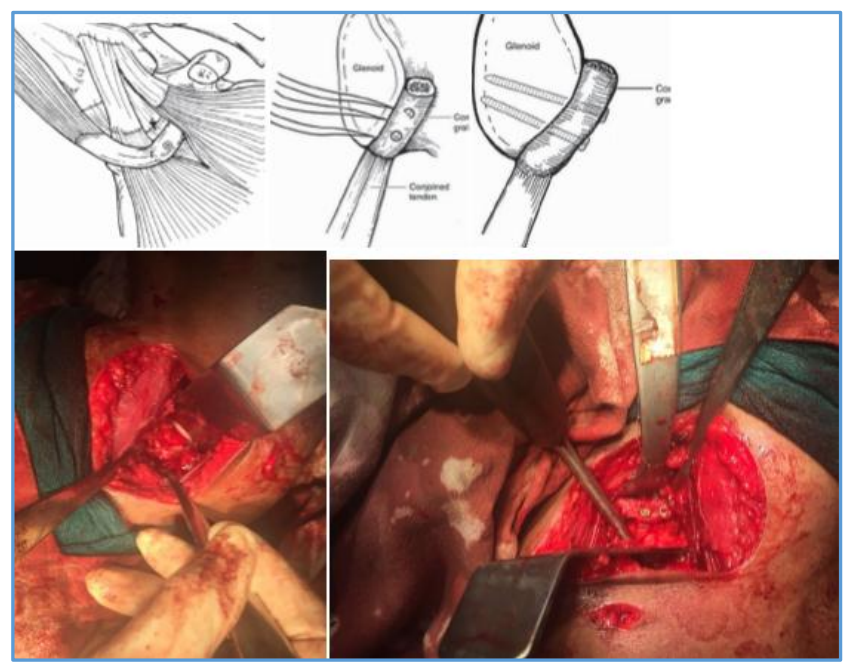

\section{Postoperative Protocol}

- $1^{\text {st }}$ Eot $-2^{\text {nd }}$ pod.

- $\quad 2^{\text {nd }}, 3^{\text {rd }}$ Eot $-5^{\text {th }}, 7^{\text {th }}$ pod.

- $\quad$ Suture removal - $10^{\text {th }}$ pod.

- Arms immobilised in a brace in internal rotation for 4 weeks.

- Pendulum exercise for first four weeks.

- Active assisted abduction exercise started after 4 weeks.

- Muscle strengthening exercise after 8 weeks.

- Full active daily activities after 6 months.

\section{Functional Evaluation}

- Two scoring system used-

1. Constant Score.

2. Rowe Score.

\section{Constant Score}

Is 100 point score in which 15 points for pain, 20 points for ADL (Activities of Daily Living), 40 points for ROM, 25 points for power to assess shoulder function. Constant score of 91 100 graded as excellent, 81-90 as good, 71-80 as satisfactory and $61-70$ as adequate outcome.

\section{Row Score}

Is a 100-point score in which 50 score for stability, 20 score for range of motion and 30 score for function of limb. 100-90 graded as excellent, 89-75 graded as good, 74-51 graded as fair, 50 or less graded as poor.
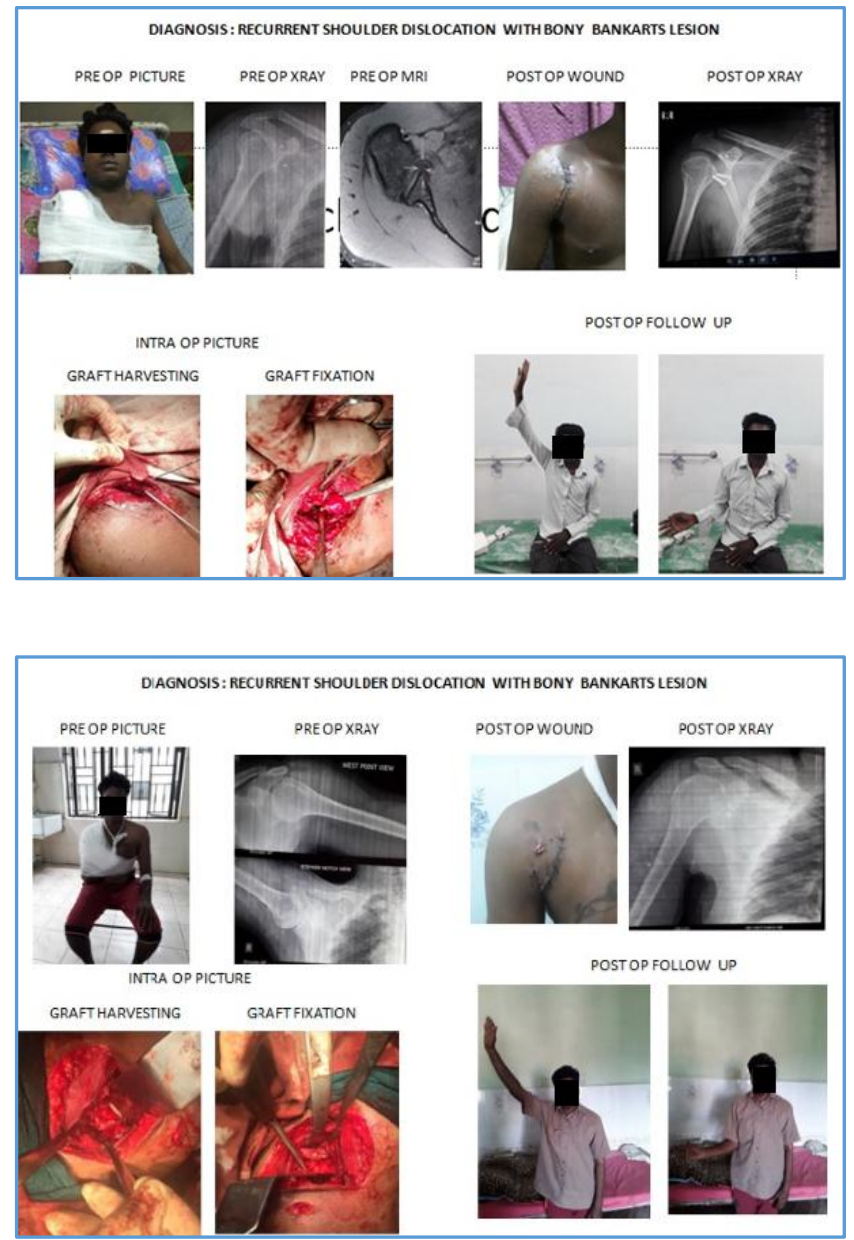


\section{RESULTS}

Total no. of recurrent shoulder dislocations with Bankart's lesion admitted in our hospital was 110 patients, of which 60 patients had Bankart's lesion. 15 patients were with $>25 \%$ bone loss, of which 10 patients were operated with this procedure.

7 patients were male and 3 female, which shows that prevalence is increased among active male individuals.

Age of the patient ranges from 24-60 years with the mean age of 28 years. Among 10 patients studied, (8) of patients were 20-40 years of age. It shows increased incidence among younger population when compared to older population.

In my study, 6 patients were affected on right side and 4 patients were on left side suggesting the right-hand dominance in the population.

Average blood loss during the procedure was $260 \mathrm{~mL}$ (range $230-300 \mathrm{~mL}$ ) and the mean operating time was 150 mins (range 115 - 165 mins).

Average follow-up period was 24 months. Osseous union of graft was achieved in all patients. According to constant scoring 6 patients had excellent outcome, 2 patients had good outcome, one patient had fair outcome. 1 patient presented with shoulder stiffness with poor outcome. None of the patients had recurrence of dislocation. Average forward flexion was 160 and External rotation was 42.2. Mean postoperative Constant-Murley score was 80. The mean Rowe score increased from 40 preoperatively to 88 at final follow-up.

Total complications 3. Two patients with surgical site complication and one patient has restricted range of motions. One patient had paraesthesia over surgical site for 5 months and recovered fully in the next follow-ups. 2 patients had superficial infection over the surgical site which was treated with antibiotics.

\section{DISCUSSION}

Hovelius L et $\mathrm{al}^{4}$ conducted long-term studies of the Latarjet procedure which have shown good-to-excellent clinical results in $86 \%$ to $97 \%$ of the patients with reported recurrent instability rates of $7 \%$ to $13.6 \%$.

Sandstrom B et al in a prospective study of 118 shoulders followed for a mean of 15.2 years. The authors reported good or excellent results in $86 \%$ of patients with $98 \%$ of patients satisfied or very satisfied with the procedure.

Yamamoto $\mathrm{N}$ et $\mathrm{al}^{5}$ concluded an osseous defect with a width that is $>$ or $=19 \%$ of the glenoid length remains unstable even after Bankart lesion repair. The stabilising mechanism of bone-grafting was required for the restoration of the glenoid concavity.

Rachbauer $\mathrm{F}$ et $\mathrm{al}^{6}$ inferenced that long-term studies of the use of iliac crest bone graft with older techniques have shown good-to-excellent results in $75 \%$ to $85 \%$ of the patients.

Warner et $\mathrm{al}^{7}$ reported on the use of tricortical iliac crest autograft for significant anterior glenoid bone defects in a series of 11 patients with recurrent instability. All grafts got incorporated and there were no cases of recurrent instability. But the use of allograft for anterior glenoid reconstruction has only been reported in small series.
Weng et $\mathrm{al}^{8}$ reported on the use of femoral head allograft in nine patients with recurrent instability and large anterior glenoid bone defects with all grafts incorporating and a mean Rowe score of 84 .

Hovelius L et $\mathrm{al}^{9}$ concluded that the overall clinical results with a satisfaction rate of $98 \%, 15$ years after the BristowLatarjet repair were as good as the results reported after any operative method for recurrent anterior shoulder dislocation.

An VV, Sivakumar BS et al ${ }^{10}$ concluded Latarjet procedure is a viable and possibly superior alternative to the Bankart repair, offering greater stability with no significant increase in complication rate.

Mizuno $\mathrm{N}$ et al ${ }^{10}$ studied that the Latarjet procedure provides excellent long-term outcomes in the treatment of recurrent anterior glenohumeral instability. Twenty years after the Latarjet procedure arthritis may develop or progress in $23.5 \%$ of cases, but the majority of arthritis is mild.

\section{Advantages}

1. A bony effect by correcting the anterior glenoid deficiency.

2. A muscular ("Sling") effect created by maintaining the inferior third, the subscapularis in an inferior position by the conjoined tendon.

3. A capsular effect by the capsular repair or repair of the coracoacromial ligament to the capsule at the end of the procedure.

Yamamoto $\mathrm{N}$ et al concluded that Latarjet technique also has been used as a bone grafting procedure to repair large glenoid defects. The "sling effect" and the "bone-block effect" have been proposed as the stabilising mechanisms of this procedure.

\section{Limitations of Study}

Since sample size is small and follow-up is only for two years, long-term outcome of this procedure could not be assessed.

\section{CONCLUSION}

Open Latarjet procedure is a reliable option for the surgical management of recurrent anterior glenohumeral instability in the setting of osseous deficiencies, greater than $25 \%$ of the glenoid surface area with good mobility and stability and may also be considered for the primary treatment of recurrent instability in which soft-tissue reconstruction is not a reasonable option.

\section{REFERENCES}

[1] Boileau P, Villalba M, Hery JY, et al. Risk factors for recurrence of shoulder instability after arthroscopic Bankart repair. J Bone Joint Surg Am 2006;88(8):1755-63.

[2] Bishop JY, Jones GL, Rerko MA, et al. 3-D CT is the most reliable imaging modality when quantifying glenoid bone loss. Clin Orthop Relat Res 2013;471(4):1251-6.

[3] Itoi E, Lee SB, Berglund LJ, et al. The effect of a glenoid defect on anteroinferior stability of the shoulder after bankart repair: a cadaveric study. J Bone Joint Surg Am 2000;82(1):35-46. 
[4] Hovelius L, Sandstrom B, Sundgren K, et al. One hundred eighteen Bristow-Latarjet repairs for recurrent anterior dislocation of the shoulder prospectively followed for fifteen years: study I-clinical results. J Shoulder Elbow Surg 2004;13(5):509-16.

[5] Yamamoto N, Muraki T, Sperling JW, et al. Stabilizing mechanism in bone-grafting of a large glenoid defect. J Bone Joint Surg Am 2010;92(11):2059-66.

[6] Rachbauer F, Ogon M, Wimmer C, et al. Glenohumeral osteoarthrosis after the Eden-Hybbinette procedure. Clin Orthop Relat Res 2000;373:135-40.

[7] Warner JJ, Gill TJ, O'hollerhan JD, et al. Anatomical glenoid reconstruction for recurrent anterior glenohumeral instability with glenoid deficiency using an autogenous tricortical iliac crest bone graft. Am J Sports Med 2006;34(2):205-12.
[8] Weng PW, Shen HC, Lee HH, et al. Open reconstruction of large bony glenoid erosion with allogeneic bone graft for recurrent anterior shoulder dislocation. Am J Sports Med 2009;37(9):1792-7.

[9] An VV, Sivakumar BS, Phan K, et al. A systematic review and meta-analysis of clinical and patientreported outcomes following two procedures for recurrent traumatic anterior instability of the shoulder: Latarjet procedure vs. Bankart repair. J Shoulder Elbow Surg 2016;25(5):853-63.

[10] Mizuno N, Denard PJ, Raiss P, et al. Long-term results of the Latarjet procedure for anterior instability of the shoulder. J Shoulder Elbow Surg 2014;23(11):1691-9. 